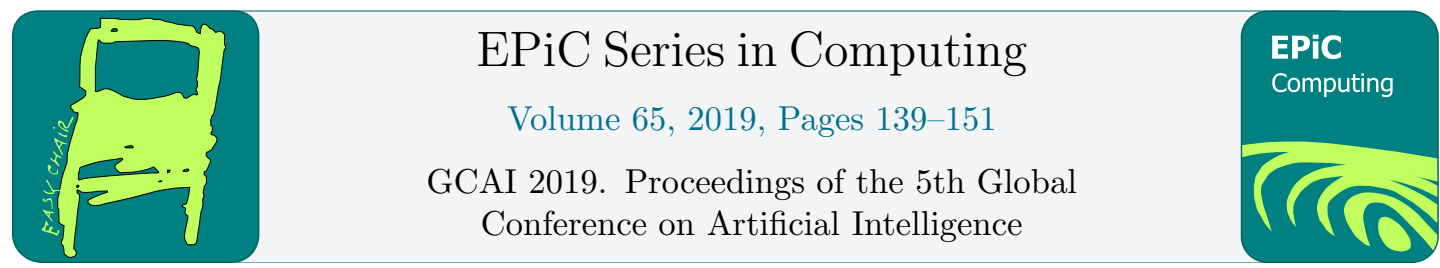

\title{
Common Knowledge in an Epistemic Logic with Hypotheses*
}

\author{
Levan Uridia ${ }^{1}$ and Dirk Walther ${ }^{2}$ \\ 1 TSU Razmadze Mathematical Institute, Tbilisi, Georgia \\ 1.uridia@freeuni.edu.ge \\ 2 DNV GL, Norway \\ dirk. walther@dnvgl.com
}

\begin{abstract}
We recall the epistemic logic $S 5^{r}$ for reasoning about knowledge under hypotheses and we investigate the extension of the logic with an operator for common knowledge. The logic $S 5^{r}$ is equipped with a modal operator of necessity that can be parameterized with hypotheses representing background assumptions while the extension with the common knowledge operator enables us to describe and reason about common knowledge among agents with possibly different background assumptions. We present an axiomatization of the logic and prove Kripke completeness and decidability results.
\end{abstract}

\section{Introduction}

We revisit the epistemic logic for reasoning about knowledge under hypotheses from [13]. The resulting logic $\mathrm{S}^{r}$ is an extension of the epistemic logic S5 with a modal operator '[.]' that can be parameterized with a hypothesis. The operator can be described as relative necessity, a notion already used by Chellas to describe conditionality [5]. It turns out that $\mathrm{S}^{r}$ is a special case of Chellas' Condition Logic. The modality ' $[\varphi]$ ' represents the knowledge state under the hypothesis $\varphi$. The formula $[\varphi] \psi$ states that 'under the hypothesis $\varphi$, the agent knows $\psi$ '. If $\varphi$ happens to be true at the current world and the agent knows that $\varphi$ implies $\psi$, then the agent knows $\psi$; otherwise, i.e., if $\varphi$ does not hold at the current world, the agent knows only what it would know anyway, i.e. without any assumptions. For instance, consider a simple dice game, where the game is won if, and only if, a three or a six has been rolled. The formula ' $[T]$ (three $\vee \operatorname{six} \leftrightarrow$ win)' states that the agent knows this rule. The parameter ' $T$ ' of the boxmodality stands for the fact that no hypothesis is being adopted by the agent. Suppose that the dice is rolled under a cup, so that the rolled number of points is concealed from the agent. Consequently, as long as the dice remains concealed, the agent does not know whether or not the game is won. This can be described by the formula ' $\neg[T]$ win'. However, the agent knows that the game is won under the hypothesis that a six has been rolled: '[ six] win'. We can

${ }^{*}$ The first author was partially supported by Shota Rustaveli National Science Foundation of Georgia (SRNSFG) grant number YS17-71.

D. Calvanese and L. Iocchi (eds.), GCAI 2019 (EPiC Series in Computing, vol. 65), pp. 139-151 
distinguish two situations: one, where the hypothesis is correct, i.e., a six has been rolled; and another one, where it is false, i.e., the dice shows a number between one to five. In the former situation, the game is won and we have that the formula holds true. In case the hypothesis is in fact wrong, the formula is not necessarily true. Irrespective of the hypotheses held by the agent, the game may still be won provided that a three has been rolled. In all other cases, the game is lost. This is different to ordinary implications, which are true whenever the premise is false or the consequent is true.

Sentences in English of the form "If $A$, then $B$." are called conditional sentences. Here, $A$ is called the antecedent (or condition) and $B$ the consequent. Conditional sentences are traditionally put into different categories (according to mood or tense) such as indicative/subjunctive or factual/counterfactual. However, there is much disagreement on the logical theory of conditional sentences (in particular that of defeasible conditionals). One logical formalization is Conditional Logic, which essentially is Propositional Logic extended with a binary operator ' $\Rightarrow$ ' standing for conditionality. Several readings of ' $\Rightarrow$ ' were proposed, among them counterfactual conditional, non-monotonic consequence relation, normality and belief revision. Historically several logical accounts of conditionals have been suggested, among them Stalnaker [12], Lewis [10] and Chellas [5].

The logic $\mathrm{S}^{r}$ rejects the common assumption that logics allow to conclude anything from false premises. We borrow the term 'explosive' from Paraconsistent Logic, but we refer to conditional operators instead of the logical consequence relation. We say that a conditional operator ' $X$ ' is explosive if the conditional $\varphi X \psi$ holds for all conclusions $\psi$ whenever the antecedent $\varphi$ is false. In this sense, implication of Classical Logic and even of Intuitionistic Logic is explosive, so is the conditional operator ' $\Rightarrow$ ' of Conditional Logic $[10,12]$. On the other hand, the relativized necessity of our logic, which is a special case of Chellas' conditional operator [5], [6], is not explosive. We have that $[\perp] \psi$ is true iff $\psi$ is universally true. The consequence relation of $\mathrm{S}^{r}$ is not paraconsistent. In this paper, we continue our investigation of $\mathrm{S}^{r}$. We present an axiomatization of $\mathrm{S}^{r}$ and its extension with the common knowledge operator in Kripke semantics.

The paper is organized as follows. In the next section, we briefly recall basic definitions on modal logic, including a modal language and the Kripke semantics, and its standard translation into first-order logic. We review a technique from [14] for obtaining Kripke completeness results for certain extensions of a modal logic. In Section 3, we recall the logic $\mathrm{S}^{r}$. In Section 4, we show that the technique from section 2 is applicable to $\mathrm{S}^{2} \mathrm{C}^{r}$ and thus prove the main result of the paper. Finally, the paper closes with a summary and outlook in Section 5.

\section{Preliminaries}

In this section, we briefly review modal logic, cf. [4]. Moreover, we introduce two techniques for obtaining completeness results: one for obtaining Kripke completeness for the extensions/enrichments of Kripke complete logics, and the other for obtaining topological completeness from Kripke completeness. The first technique is introduced in detail in [14], whereas the second is well known in the literature [3,2].

Let $\langle\Pi, M\rangle$ be a signature consisting of countable sets $\Pi$ and $M$ of symbols for propositions and modalities, respectively. The propositional modal language $\mathcal{L}$ for this signature consists of formulas $\varphi$ that are built up inductively according to the grammar:

$$
\varphi::=p|\neg \varphi| \varphi \wedge \varphi \mid \square_{m} \varphi,
$$

where $p$ ranges over proposition symbols in $\Pi$ and $m$ over modality symbols in $M$. The logical 
symbols ' $T$ ' and ' $\perp$ ', and the additional connectives such as ' $V$ ', ' $\rightarrow$ ' and ' $\leftrightarrow$ ' and the dual modalities ' $\diamond_{m}$ ' with $m \in M$ are defined as usual, i.e.: $\top:=p \vee \neg p$ for some atomic proposition $p ; \perp:=\neg \top ; \varphi \vee \psi:=\neg(\neg \varphi \wedge \neg \psi) ; \varphi \rightarrow \psi:=\neg \varphi \vee \psi ; \varphi \leftrightarrow \psi:=(\varphi \rightarrow \psi) \wedge(\psi \rightarrow \varphi)$; and $\diamond_{m} \varphi:=\neg \square_{m} \neg \varphi$.

A subset $L$ of the propositional modal language $\mathcal{L}$ is a modal logic iff it contains all propositional tautologies, is closed under substitution, modus ponens and modal replacement (MREP) $\frac{p \leftrightarrow q}{\square_{m} p \leftrightarrow \square_{m} q}$, for $m \in M$. The modal logic $L$ is called normal if it contains the formulas (K) $\square_{m}(p \rightarrow q) \rightarrow\left(\square_{m} p \rightarrow \square_{m} q\right)$ and are closed under (NEC) $\frac{p}{\square_{m} p}$. The smallest normal modal logic is commonly denoted with $\mathrm{K}$.

\subsection{Kripke Semantics}

The relational semantics for the propositional modal language $\mathcal{L}$ is based on Kripke structures for the signature $\langle\Pi, M\rangle$ of $\mathcal{L}$. Formally, an $M$-frame (or Kripke frame) is a tuple $\mathfrak{F}=$ $\left(W,\left\{R_{m}\right\}_{m \in M}\right)$, where $W$ is a non-empty set of worlds and $R_{m} \subseteq W^{2}$ a binary relation over $W$, for every $m \in M$. A Kripke model for $\langle\Pi, M\rangle$ is a pair $\mathfrak{M}=(\mathfrak{F}, V)$ consisting of a Kripke frame $\mathfrak{F}=\left(W,\left\{R_{m}\right\}_{m \in M}\right)$ together with a valuation function $V: \Pi \rightarrow 2^{W}$ assigning to every proposition $p$ in $\Pi$ a set $V(p)$ of worlds. A Kripke model $\mathfrak{M}=(\mathfrak{F}, V)$ is said to be based on the frame $\mathfrak{F}$.

An interpretation of formulas from $\mathcal{L}$ is given by means of a satisfaction relation ' $\models$ ', which is a binary relation between pointed models and formulas. A pointed model is a pair $\langle\mathfrak{M}, w\rangle$, where $\mathfrak{M}=\left(W,\left\{R_{m}\right\}_{m \in M}, V\right)$ is a Kripke model and $w$ a world from $W$. The satisfaction relation is defined inductively on the structure of formulas $\varphi$ as:

- $\langle\mathfrak{M}, w\rangle \models p$ iff $w \in V(p)$;

- $\langle\mathfrak{M}, w\rangle \models \neg \psi$ iff $\langle\mathfrak{M}, w\rangle \not \models \psi ;$

- $\langle\mathfrak{M}, w\rangle \models \psi \wedge \chi$ iff $\langle\mathfrak{M}, w\rangle \models \psi$ and $\langle\mathfrak{M}, w\rangle \models \chi$;

- $\langle\mathfrak{M}, w\rangle \models \square_{m} \psi$ iff for all $v \in W$ with $(w, v) \in R_{m},\langle\mathfrak{M}, v\rangle \models \psi$.

A formula $\varphi$ is said to be true at $w$ in $\mathfrak{M}$ iff $\langle\mathfrak{M}, w\rangle \models \varphi ; \varphi$ is satisfiable iff there is a pointed model $\langle\mathfrak{M}, w\rangle$ at which it is true; $\varphi$ is valid in $\mathfrak{M}$ (written ' $\mathfrak{M} \models \varphi$ ') iff $\langle\mathfrak{M}, w\rangle \models \varphi$ for all $w$ in $\mathfrak{M} ; \varphi$ is valid on $\mathfrak{F}$ (written ' $\mathfrak{F}=\varphi^{\prime}$ ) iff $\varphi$ is valid in all models based on $\mathfrak{F}$; and $\varphi$ is valid in the class $\mathcal{C}$ of Kripke frames (written ' $\models_{\mathcal{C}} \varphi$ ') iff it is valid in every Kripke frame from $\mathcal{C}$.

The set of $\mathcal{L}$-formulas that are valid in a class $\mathcal{C}$ of Kripke frames is called the $\mathcal{L}$-theory $\operatorname{Th}_{\mathcal{L}}(\mathcal{C})$ of $\mathcal{C}$, i.e.: $\operatorname{Th}_{\mathcal{L}}(\mathcal{C}):=\{\varphi \in \mathcal{L} \mid$ for every $\mathfrak{F}$ from $\mathcal{C}, \varphi$ is valid in $\mathfrak{F}\}$.

A modal logic $L$ is said to be Kripke complete w.r.t. $\mathcal{C}$ iff $L \supseteq \mathrm{Th}_{\mathcal{L}}(\mathcal{C})$, and $L$ is said to be sound w.r.t. $\mathcal{C}$ iff $L \subseteq \operatorname{Th}_{\mathcal{L}}(\mathcal{C})$.

\subsection{Standard Translation}

The relationship of a modal logic to first-order logic is made precise by the so-called standard translation $\operatorname{ST}(\cdot)$, which assigns to a modal formula $\varphi$ a corresponding first-order formula $\operatorname{ST}_{x}(\varphi)$ with one free variable $x$. The signature of the first-order language contains unary predicate symbols $P$ and binary predicate symbols $R_{m}$, one $P$ for every $p \in \Pi$ and one $R_{m}$ for every 
$m \in M$. The translation function $\operatorname{ST}(\cdot)$ is inductively defined as follows:

$$
\begin{aligned}
\operatorname{ST}_{x}(p) & :=P(x) \\
\operatorname{ST}_{x}(\neg \varphi) & :=\neg \mathrm{ST}_{x}(\varphi) \\
\operatorname{ST}_{x}(\varphi \wedge \psi) & :=\operatorname{ST}_{x}(\varphi) \wedge \operatorname{ST}_{x}(\psi) \\
\operatorname{ST}_{x}\left(\square_{m} \varphi\right) & :=\forall y\left(R_{m}(x, y) \rightarrow \operatorname{ST}_{y}(\varphi)\right)
\end{aligned}
$$

where $y$ is a fresh variable for every occurrence of a box-modality.

A Kripke structure $\mathfrak{M}=\left(W,\left\{R_{m}\right\}_{m \in M}, V\right)$ for $\langle\Pi, M\rangle$ can be seen as a first-order structure interpreting the formula $\operatorname{ST}_{x}(\varphi)$. While a predicate symbol $R_{m}$ is interpreted using the binary relation $R_{m}$ over $W$ that is interpreting the modality $m$ in $M$, a predicate symbol $P$ is interpreted as the subset $V(p)$ of $W$, where $p$ is the proposition symbol from $\Pi$ that corresponds to $P$. Neither constants nor function symbols are introduced by the standard translation. In the first-order structure $\mathfrak{M}$, however, we introduce a dedicated constant $c_{w}$ for every world $w \in W$ and we interpret $c_{w}$ as $w$. At the level of pointed models $\langle\mathfrak{M}, w\rangle$, the relationship between $\varphi$ and $\operatorname{sT}_{x}(\varphi)$ is such that:

$$
\langle\mathfrak{M}, w\rangle \models \varphi \text { iff } \mathfrak{M} \models \operatorname{ST}_{x}(\varphi)\left[x \mapsto c_{w}\right],
$$

where $\left[x \mapsto c_{w}\right]$ substitutes every occurrence of the free variable $x$ in $\operatorname{ST}_{x}(\varphi)$ with the constant $c_{w}$. Note that $\operatorname{ST}_{x}(\varphi)\left[x \mapsto c_{w}\right]$ is a sentence, i.e. a first-order formula without free variables.

When considering the notion of validity on frames $\mathfrak{F}$, we have that $\varphi$ corresponds to the monadic second-order formula $\forall \vec{P} \forall x \operatorname{ST}_{x}(\varphi)$ as follows:

$$
\mathfrak{F} \models \varphi(\vec{p}) \text { iff } \mathfrak{F} \models \forall \vec{P} \forall x \operatorname{ST}_{x}(\varphi),
$$

where $\vec{p}$ are the propositions from $\Pi$ that occur in $\varphi$, and $\vec{P}$ are the corresponding unary predicates.

\subsection{Completeness by Modal Definitions}

In [14] we introduced a technique on how to obtain Kripke completeness w.r.t. a specific class of Kripke structures for certain extensions of complete modal logics. We apply this technique to extensions of the modal logic S5.

By extending a modal logic $L$ with a formula $\varphi$ we mean obtaining a modal logic $L^{\prime}$ as a set of formulas that is minimal w.r.t. $\subseteq$, that contains all tautologies over the symbols for propositions occurring in $L \cup\{\varphi\}$, that contains all formulas from $L \cup\{\varphi\}$ and that is closed under substitution, modus-ponens and modal replacement. In general, $L \cup\{\varphi\}$ is not a modal logic as adding $\varphi$ to $L$ does not necessarily satisfy the conditions above. Moreover, an extension of a modal logic, which is Kripke complete w.r.t. a class $\mathcal{C}$ of models, is not necessarily complete w.r.t. $\mathcal{C}$ itself nor any other class of models. See to [14] for further details and examples. We are interested in studying formulas of a specific form (modal definitions) that, when used to extend a modal logic, yield a modal logic that is complete w.r.t. a specific class of models. Let $\mathcal{L}$ be a propositional modal language over the signature $\langle\Pi, M\rangle$. Let $\varphi(\vec{p})$ be a formula in $\mathcal{L}$, where $\vec{p}$ are the propositions occurring in $\varphi$. Let ' + ' be a fresh symbol for a unary modality not in $M$, and ' $\boxplus$ ' the box-version of this modality. A modal definition in $\mathcal{L}$ is a formula of the form

$$
\boxplus p \leftrightarrow \varphi(\vec{p}),
$$


where $\vec{p}$ contains $p$. The box-modality ' $\boxplus$ ' is defined in terms of a modal formula in which ' $\boxplus$ ' does not occur. Notice that the modal definition $\boxplus p \leftrightarrow \varphi(\vec{p})$ itself is a formula in the propositional modal language over the extended signature $\langle\Pi, M \cup\{+\}\rangle$.

In this paper, we only consider modal definitions of the form $\boxplus p \leftrightarrow \varphi(\vec{p})$, where the boxmodality ' $\boxplus$ ' does not occur in $\varphi(\vec{p})$ and the symbol ' + ' stands for a unary modality.

A modal definition is interpreted in models $\mathfrak{M}=(\mathfrak{F}, V)$ that are based on $M \cup\{+\}$-frames $\mathfrak{F}=\left(W,\left\{R_{m}\right\}_{m \in M} \cup\left\{R_{+}\right\}\right)$, i.e., frames that are extended with a binary relation $R_{+}$to interpret the new box-modality ' $\boxplus$ '. The semantics of ' $\boxplus$ ' can be defined in the usual way as for any other box-modality:

- $\langle\mathfrak{M}, w\rangle \models \boxplus \psi$ iff for all $v \in W$ with $(w, v) \in R_{+}$, it holds that $\langle\mathfrak{M}, v\rangle \models \psi$.

We want to interpret ' $⿴ 囗 十$ ' as specified in the modal logic $L^{\prime}$ obtained from the modal logic $L$ extended with a modal definition of ' $⿴ 囗 十$ '. To this end, we have to confine ourselves to the models from $\mathcal{C}\left(L^{\prime}\right)$, i.e., all models from $\mathcal{K}_{\langle\Pi, M \cup\{+\}\rangle}$ in which all formulas of $L^{\prime}$ are valid. It is now interesting to investigate the relationship between the modal definition of ' $⿴ 囗 十$ ' and the properties of the relation $R_{+}$in the models from $\mathcal{C}\left(L^{\prime}\right)$.

Not all modal definitions yield a relational semantics for the logic extended with the newly defined modality. Taking the standard translation of a formula $\varphi$ that is used in a definition $\boxplus p \leftrightarrow \varphi(\vec{p})$ results in the second-order formula $\forall \vec{P} \forall x \mathrm{ST}_{x}(\varphi)$, where the predicates in $\vec{P}$ correspond to the propositional variables in $\vec{p}$. We are interested in elementary formulas, i.e., those formulas $\varphi$ for which there exists a first-order formula that is equivalent to the second-order formula $\forall \vec{P} \forall x \operatorname{ST}_{x}(\varphi)$, that additionally yield a relational semantics for the new modality ' + '. It is a non-trivial problem to give a syntactic characterization of such formulas $\varphi$ that are suitable for defining fresh modalities.

To start tackling this problem, we introduce the notion of a 'relational modal definition'.

Definition 1. Let $\mathcal{L}$ be a propositional modal language over the signature $\langle\Pi, M\rangle$. Let $\varphi(p$, $\left.p_{1}, \ldots, p_{n}\right)$ with $n \geq 0$ be a formula in $\mathcal{L}$, where $p, p_{1}, \ldots, p_{n}$ are the symbols for propositions occurring in $\varphi$. Let ' + ' be a fresh symbol for a unary modality not in $M$, and ' $\boxplus$ ' the box-version of this modality.

A modal definition $\boxplus p \leftrightarrow \varphi\left(p, p_{1}, \ldots, p_{n}\right)$ is called a relational modal definition if there exists a first-order formula $\Psi_{+}(x, y)$ with two free variables $x$ and $y$ using only predicates that occur in $\operatorname{ST}_{x}\left(\varphi\left(p, p_{1}, \ldots, p_{n}\right)\right)$ such that for every $\psi \in \mathcal{L}$, it holds that for all pointed models $\langle\mathfrak{M}, w\rangle$, $\mathfrak{M}=\left((\forall y)\left(\Psi_{+}(x, y) \rightarrow \operatorname{ST}_{y}(\psi)\right)\right)\left[x \mapsto c_{w}\right]$ iff $\mathfrak{M} \models \operatorname{ST}_{x}\left(\varphi\left(\psi, p_{1}, \ldots, p_{n}\right)\right)\left[x \mapsto c_{w}\right]$.

Example 1. Let us consider modal logic $K$ extended with a new modality ' $\boxplus$ '. A formula $\boxplus p \leftrightarrow \square p \wedge p$ is a relational modal definition. Indeed, for every pointed model $\langle\mathfrak{M}, w\rangle$, it holds that $\mathfrak{M}=((\forall y)(x R y \rightarrow P(y)) \wedge P(x)))\left[x \mapsto c_{w}\right]$ iff $((\forall y)(\Psi(x, y) \rightarrow P(y)))\left[x \mapsto c_{w}\right]$, where $\Psi(x, y)$ is the formula $(x R y) \vee(x=y)$.

We note that elementarity is neither a sufficient nor a necessary condition for modal formulas being suitable for a relational modal definition; see, e.g., the reduction axiom for $\mathrm{S}^{r}$ in the following section which yields a relational modal definition despite it being non-elementary.

Let $\Psi_{+}(x, y)$ be the first-order formula with two free variables $x$ and $y$ corresponding to a relational modal definition. Given a model $\mathfrak{M}=(\mathfrak{F}, V)$ with $\mathfrak{F}=\left(W,\left\{R_{m}\right\}_{m \in M}\right)$, we uniquely construct the model $\mathfrak{M}_{+}=\left(\mathfrak{F}_{+}, V\right)$, where the underlying frame $\mathfrak{F}_{+}$is obtained from $\mathfrak{F}$ by adding the binary relation $R_{+} \subseteq W \times W$ defined as:

$$
(v, w) \in R_{+} \text {iff } \mathfrak{M} \models \Psi_{+}(x, y)\left[x \mapsto c_{v}, y \mapsto c_{w}\right] .
$$


For a class $\mathcal{C}$ of models, we denote with $\mathcal{C}_{+}$the class consisting of the models $\mathfrak{M}_{+}$, where $\mathfrak{M}$ ranges over the models in $\mathcal{C}$.

Formulas from the extended language $\mathcal{L}_{+}$can be translated to formulas in $\mathcal{L}$ in a straightforward way.

Definition 2. Let $\mathcal{L}$ and $\mathcal{L}_{+}$be propositional modal languages over the signatures $\langle\Pi, M\rangle$ and $\langle\Pi, M \cup\{+\}\rangle$, respectively, where ' + ' is a fresh symbol for a unary modality not in $M$, and ' $\boxplus$ ' the box-version of this modality. The translation function ${ }^{*}: \mathcal{L}^{+} \rightarrow \mathcal{L}$ for the modal definition $\boxplus p \leftrightarrow \varphi_{+}\left(p, p_{1}, \ldots, p_{n}\right)$ is inductively defined as follows, where $m$ ranges over $M: p^{*}:=p$, $(\varphi \vee \psi)^{*}:=\varphi^{*} \vee \psi^{*},(\neg \varphi)^{*}:=\neg \varphi^{*},\left(\square_{m} \varphi\right)^{*}:=\square_{m} \varphi^{*},(\boxplus \psi)^{*}:=\varphi_{+}\left(\psi^{*}, p_{1}, \ldots, p_{n}\right)$.

The following theorem shows the intended completeness technique.

Theorem 1 ([14]). Let $\mathcal{L}$ and $\mathcal{L}_{+}$be propositional modal languages over the signatures $\langle\Pi, M\rangle$ and $\langle\Pi, M \cup\{+\}\rangle$, respectively, where ' + ' is a fresh symbol for a unary modality not in $M$, and ' $\boxplus$ ' the box-version of this modality. Let $L \subseteq \mathcal{L}$ be a normal modal logic that is sound and complete w.r.t. some class $\mathcal{F}$ of Kripke frames. Obtain $L_{+} \subseteq \mathcal{L}_{+}$from $L$ by adding a relational modal definition $\boxplus p \leftrightarrow \varphi\left(p_{1}, \ldots, p_{n}\right)$ as the only axiom schema for ' $\boxplus$ '. Then the logic $L_{+}$is sound and complete w.r.t. the class $\mathcal{F}_{+}$.

\section{The Modal Logic S5 ${ }^{r}$}

In this section, we recall the multi-modal logic $S 5^{r}$ from [13] together with the completeness result w.r.t. a particular class of models called basic structures.

\subsection{Syntax}

The language of $\mathrm{S}^{r}$ is the language of propositional logic extended with modal operators parameterized with $\mathrm{S}^{r}$-formulas. Formally, this is done as follows. Let $\Pi$ be a countable set of propositions. Formulas $\varphi$ of the language $\mathcal{L}$ are defined inductively over $\Pi$ by the following grammar:

$$
\varphi, \psi::=p|\neg \varphi| \varphi \vee \psi \mid[\varphi]_{K} \psi,
$$

where $p$ ranges over propositions in $\Pi$. The logical symbols ' $T$ ' and ' $\perp$ ', and additional operators such as ' $\wedge$ ', ' $\rightarrow$ ', ' $\leftrightarrow$ ', and the dual modalities ' $\langle\varphi\rangle_{K}$ ' are defined as usual.

\subsection{Relational semantics}

Modal formulas are commonly evaluated in models containing a binary relation over the domain, one for each modality in the modal language. In this case, however, every binary relation is determined by the valuation of the atomic propositions in the domain. Therefore, it is sufficient to consider models without relations, which we call basic structures. Formally, a basic structure $\mathfrak{M}$ is a tuple $\mathfrak{M}=(W, V)$, where $W$ is a non-empty set of worlds and $V: \Pi \rightarrow 2^{W}$ a valuation function mapping every atomic proposition $p$ to a set of worlds $V(p)$ at which it is true. The relations that are required to evaluate the modalities are defined alongside the satisfaction relation. But first we introduce an auxiliary notion, a binary operation ' $\otimes$ ' on sets yielding a binary relation. Let $X$ and $Y$ be two sets. Let $X \otimes Y$ be a binary relation over $X \cup Y$ such that

$$
X \otimes Y=X^{2} \cup(X \times Y) \cup Y^{2} .
$$


We are now ready to introduce the semantics of $\mathrm{S}^{r}$. It differs from the semantics of Public Announcement Logic $[11,16]$ in that the model does not change during the evaluation of formulas.

Let $\mathfrak{M}=(W, V)$ be a basic structure. The logical satisfaction relation ' $=$ ' is defined by induction on the structure of $\mathrm{S}^{r}$-formulas as follows: For all $p \in \Pi$ and all $\varphi, \psi \in \mathcal{L}$,

- $\langle\mathfrak{M}, w\rangle \models p$ iff $w \in V(p)$;

- $\langle\mathfrak{M}, w\rangle \models \varphi \vee \psi$ iff $\langle\mathfrak{M}, w\rangle \models \varphi$ or $\langle\mathfrak{M}, w\rangle \models \psi$;

- $\langle\mathfrak{M}, w\rangle \models \neg \varphi$ iff $\langle\mathfrak{M}, w\rangle \not \models \varphi$;

- $\langle\mathfrak{M}, w\rangle \models[\varphi]_{K} \psi$ iff for all $v \in W$ with $(w, v) \in R_{\varphi}$, it holds that $\langle\mathfrak{M}, v\rangle \models \psi$;

where $R_{\varphi}=\left(W \backslash \llbracket \varphi \rrbracket_{\mathfrak{M}}\right) \otimes \llbracket \varphi \rrbracket_{\mathfrak{M}}$ as defined in Equation (1) and $\llbracket \varphi \rrbracket_{\mathfrak{M}}=\{w \in W \mid\langle\mathfrak{M}, w\rangle \models \varphi\}$ is the extension of $\varphi$ in $\mathfrak{M}$. We say that a $\mathrm{S5}^{r}$-formula $\varphi$ is satisfiable if there is a model $\mathfrak{M}$ and a world $w$ in $\mathfrak{M}$ such that $\langle\mathfrak{M}, w\rangle \models \varphi ; \varphi$ is valid in $\mathfrak{M}$ if $\langle\mathfrak{M}, w\rangle \models \varphi$ for all $w$ in $\mathfrak{M}$; and $\varphi$ is valid if $\varphi$ is valid in all models. We will refer to the relation $R_{\varphi}$ as being determined by $\varphi$ and a model.

According to the semantics, a formula determines a binary relation in a model. The following proposition states the properties of such relations.

Proposition 1. Let $\varphi$ be an $S 5^{r}$-formula and let $\mathfrak{M}=(W, V)$ be a basic structure. Then, the relation $R_{\varphi}$ determined by $\varphi$ and $\mathfrak{M}$ is a one-step total preorder, i.e., $R_{\varphi}$ satisfies the following conditions:

- $R_{\varphi}$ is transitive: $\forall x y z\left(R_{\varphi}(x, y) \wedge R_{\varphi}(y, z) \rightarrow R_{\varphi}(x, z)\right)$;

- $R_{\varphi}$ is total: $\forall x y\left(R_{\varphi}(x, y) \vee R_{\varphi}(y, x)\right)$; and

- $R_{\varphi}$ is one-step: $\forall x y z\left(\left(R_{\varphi}(x, y) \wedge \neg R_{\varphi}(y, x) \wedge R_{\varphi}(x, z)\right) \rightarrow R_{\varphi}(z, y)\right)$.

Instead of 'preorder' also the term 'quasiorder' is often used in the literature. Note that totality implies reflexivity and that a symmetric total preorder is an equivalence relation. The proposition is readily checked as any relation $R_{\varphi}$ in a model determined by $\varphi$ is defined using the operation ' $\otimes$ ', which always yields a so-called 'one-step total preorder'. As the domain of a model is non-empty, it contains at least one point and, thus, the smallest relation $R_{\varphi}$ is the edge of a single reflexive point.

Proposition 2. The relation $R_{\varphi}$ for every formula $\varphi \in S 5^{r}$ is characterized by the following condition: $R_{\varphi}(w, v)$ iff $w \in \llbracket \varphi \rrbracket$ implies that $v \in \llbracket \varphi \rrbracket$.

Figure 1 illustrates the relation $R_{\varphi}$ in a model $\mathfrak{M}$. The domain of $\mathfrak{M}$ is partitioned into two clusters, the worlds in each of which are fully connected (reflexive and symmetric edges within the clusters are not shown). Between the clusters there are outgoing directed edges from worlds in the cluster on the left- to worlds in the cluster on the right-hand side, but not vice versa.

Consider the following example, which illustrates the effect that hypotheses can have on an agent's knowledge.

Example 2. Let $\mathfrak{M}=(W, V)$ be a basic structure with $W=\{x, y\}, V\left(p_{h}\right)=V\left(p_{c}\right)=\{x\}$ and $V\left(p_{u}\right)=\{x, y\}$. Intuitively, the three propositions $p_{h}, p_{c}$ and $p_{u}$ stand for hypothesis, conclusion and universal or already established knowledge, respectively. Then, $\left[p_{h}\right]_{K} p_{u}$ is true at $x$ and $y$ in $\mathfrak{M}$. In fact, we have that $\langle\mathfrak{M}, x\rangle=[\varphi]_{K} p_{u}$ for every $S 5^{r}$-formula $\varphi$, because $p_{u}$ holds everywhere in $\mathfrak{M}$. But $\left[p_{h}\right]_{K} p_{c}$ holds only at $x$ and not at $y$, because $\langle\mathfrak{M}, x\rangle \models p_{h}$ and $p_{h}$ implies $p_{c}$ everywhere in $\mathfrak{M}$. 


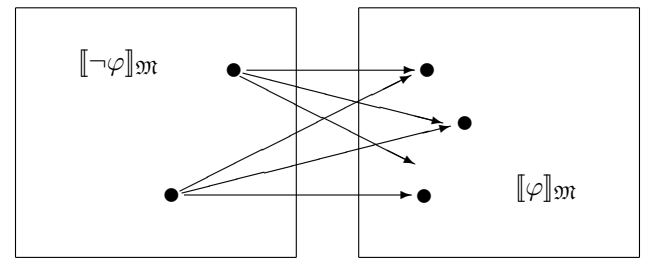

Figure 1: Model $\mathfrak{M}$ with Relation $R_{\varphi}$

\subsection{Axiomatization}

We now present a sound and complete axiomatization of $\mathrm{S}^{r}$ from [13]. The axiom system consists of all propositional tautologies and the following axioms:

(K) $[\top]_{K}(p \rightarrow q) \rightarrow\left([\top]_{K} p \rightarrow[\top]_{K} q\right)$

( $\mathrm{T})[\top]_{K} p \rightarrow p$

(4) $[\top]_{K} p \rightarrow[T]_{K}[\top]_{K} p$

(B) $p \rightarrow[T]_{K} \neg[\top]_{K} \neg p$

(R) $[\varphi]_{K} p \leftrightarrow[\top]_{K} p \vee\left(\varphi \wedge[\top]_{K}(\varphi \rightarrow p)\right)$

The first four axioms are similar to the axioms known from the modal epistemic logic S5 characterizing any modality $[\varphi]_{K}$ in our logic $S 5^{r}$ as epistemic operator that can be used to represent what is known under the hypothesis $\varphi$.

The axioms ( $\mathrm{T}),(4)$, and (B) are for the modality $[\mathrm{T}]_{K}$ only, whereas we need additional instances of the axioms $(\mathrm{K})$ and $(\mathrm{R})$, namely the ones for each modal parameter $\varphi$ (cf. Section 3.1). The reduction axiom (R) states that every modality $[\varphi]_{K}$ is definable in terms of the basic modal operator $[T]_{K}$, which corresponds to the S5-box or the universal modality. As it was already mentioned in the introduction, Axiom (R) corresponds to the definition of the modal operator 'Modest Enrichment (Type B)' in [7].

Theorem 2 ([13]). The system $S^{r}$ is sound and complete w.r.t. the class of basic structures.

\section{The Modal Logic S5C ${ }^{r}$}

Probably one of the most interesting examples in the direction of dynamic epistemic logic is provided by the notion of common knowledge. Common knowledge, originally defined by Lewis [9], has been extensively studied from various perspectives including philosophy [1], game theory [15], artificial intelligence [8] and modal logic. The usual definition of common knowledge of agents is given as an infinite conjunction of nested individual knowledge. For example in the case of two agents, say, Agent 1 and Agent 2 with corresponding knowledge operators $\square_{1}$ and $\square_{2}$, common knowledge that $p$ is formalized by an infinite formula of the form $\varphi \wedge \square_{1} \varphi \wedge$ $\square_{2} \varphi \wedge \square_{1} \square_{1} \varphi \wedge \square_{1} \square_{2} \varphi \wedge \square_{2} \square_{1} \varphi \wedge \square_{2} \square_{2} \varphi \wedge \square_{1} \square_{1} \square_{1} \varphi \ldots$

Alternatively, we can abstract from the identity of the agents and refer to an agent by using formulas that describe the hypotheses assumed by the agent. With that in mind, it seems natural to extend the language a common knowledge operator. In this section, we extend $\mathrm{S}^{r}$ with modalities for common knowledge and prove Kripke completeness of the resulting logic. 
Definition 3 (Syntax of S5C ${ }^{r}$ ). Let $\Pi$ be a countable set of propositions. Formulas $\varphi$ of S5C are defined inductively over $\Pi$ by the following grammar:

$$
\varphi, \psi, \varphi_{i}::=p|\neg \varphi| \varphi \vee \psi\left|[\varphi]_{K} \psi\right|\left[\left\{\varphi_{1}, \ldots, \varphi_{n}\right\}\right]_{C} \psi
$$

where $p$ ranges over atomic propositions in $\Pi$ and $n \geq 0$.

To improve readability, we index the modalities with ' $\mathrm{K}$ ' and ' $\mathrm{C}$ ' to indicate that they stand for knowledge and common knowledge, respectively. Moreover, we may omit the braces in $\left[\left\{\varphi_{1}, \ldots, \varphi_{n}\right\}\right]_{C} \psi$ and simply write $\left[\varphi_{1}, \ldots, \varphi_{n}\right]_{C} \psi$. Formulas of $\mathrm{S} \mathrm{C}^{r}$ are evaluated in basic structures as well. A modality $[\Phi]_{C}$ is a necessity depending on the formulas in the set $\Phi$. The semantics of $[\Phi]_{C}$ is based on the relations $R_{\varphi}$ with $\varphi \in \Phi$ as follows.

Definition 4 (Semantics of $\left.\mathrm{S}^{2} \mathrm{C}^{r}\right)$. Let $\mathfrak{M}=(W, V)$ be a basic structure. The satisfaction

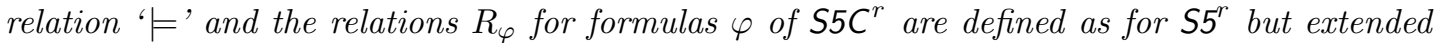
with the following clauses: For all S5C $\mathrm{C}^{r}$-formulas $\psi$ and all finite sets $\Phi$ of S5C $\mathrm{C}^{r}$-formulas,

- $\langle\mathfrak{M}, w\rangle \models[\Phi]_{C} \psi$ iff for all $v \in W$ with $(w, v) \in R_{\Phi}^{C}$, it holds that $\langle\mathfrak{M}, v\rangle \models \psi$,

where $R_{\Phi}^{C}=\left(\bigcup_{\varphi \in \Phi} R_{\varphi}\right)^{*}$ and $(\cdot)^{*}$ yields the reflexive and transitive closure of a relation.

Due to the simple structure of one-step relations, it may seem the union $\bigcup_{\varphi \in \Phi} R_{\varphi}$ is itself transitive. The following example illustrates that this is not always the case.

Example 3. Let $\mathfrak{M}=(W, V)$ be a model, where $W=\{x, y, s, t\}, V(p)=\{t, y\}$ and $V(q)=$ $\{x, t\}$. Then $R_{p}=\{s, x\} \otimes\{t, y\}$ and $R_{q}=\{s, y\} \otimes\{t, x\}$. Hence, we have that $\left(R_{p} \cup R_{q}\right)(t, y)$ and $\left(R_{p} \cup R_{q}\right)(y, s)$ but not $\left(R_{p} \cup R_{q}\right)(t, s)$; see Figure 2 .
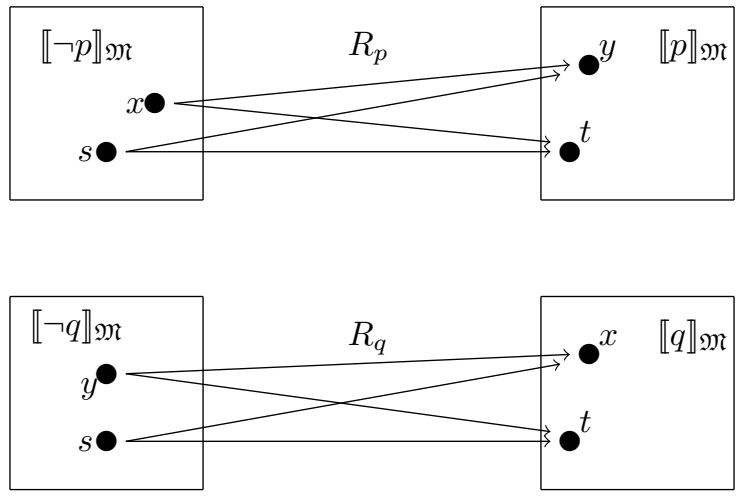

Figure 2: Model $\mathfrak{M}$ with Relations $R_{p}$ and $R_{q}$

The example also shows that the reflexive and transitive closure of the union relation $\bigcup_{\varphi \in \Phi} R_{\varphi}$ will be a cluster if for every pair $s$ and $t$ of worlds that are from different clusters of each relation $R_{\varphi}$ with $\varphi \in \Phi$, there are formulas $\theta$ and $\psi$ in $\Phi$ such that $\langle\mathfrak{M}, s\rangle \models \theta \wedge \neg \psi$ and $\langle\mathfrak{M}, t\rangle \models \neg \theta \wedge \psi$.

In the remainder of the section, we focus on the case where $\Phi=\left\{\varphi_{1}, \varphi_{2}\right\}$. It can readily be seen that the results can be generalized to an arbitrary finite subset of formulas. The definition of the satisfaction relation boils down to the following:

- $\langle\mathfrak{M}, w\rangle \models[\Phi]_{C} \psi$ iff for all $v \in W:\left(R_{\varphi_{1}} \cup R_{\varphi_{2}}\right)^{*}(w, v)$ implies that $\langle\mathfrak{M}, v\rangle \models \psi$. 
The proof of the following proposition follows from the semantics of $[\Phi]_{C} \psi$ and properties of the transitive and reflexive closure of the relation.

Proposition 3. Let $\mathfrak{M}$ be a model and $w$ be a world in $\mathfrak{M}$. Then the following holds: $\langle\mathfrak{M}, w\rangle \models$ $[\Phi]_{C} \psi$ iff $\langle\mathfrak{M}, w\rangle=\psi \wedge\left[\varphi_{1}\right]_{K} \psi \wedge\left[\varphi_{2}\right]_{K} \psi \wedge\left[\varphi_{1}\right]_{K}\left[\varphi_{2}\right]_{K} \psi \wedge\left[\varphi_{2}\right]_{K}\left[\varphi_{1}\right]_{K} \psi \wedge\left[\varphi_{1}\right]_{K}\left[\varphi_{1}\right]_{K} \psi \wedge \cdots$.

The next proposition shows that the infinitary nature of the common knowledge operator could be captured by a formula of the language $\mathrm{S}^{r}$.

Proposition 4. Let $\mathfrak{M}$ be a model and $w$ be a world in $\mathfrak{M}$. Then the following holds: $\langle\mathfrak{M}, w\rangle \models$ $\left\langle\left\{\varphi_{1}, \varphi_{2}\right\}\right\rangle_{C} \psi$ iff $\langle\mathfrak{M}, w\rangle \models\left\langle\varphi_{1}\right\rangle_{K} \psi \vee\left\langle\varphi_{2}\right\rangle_{K} \psi \vee\left\langle\varphi_{1}\right\rangle_{K}\left\langle\varphi_{2}\right\rangle_{K} \psi \vee\left\langle\varphi_{2}\right\rangle_{K}\left\langle\varphi_{1}\right\rangle_{K} \psi$.

Proof. For the direction from left to right, suppose that $\langle\mathfrak{M}, w\rangle \models\left\langle\left\{\varphi_{1}, \varphi_{2}\right\}\right\rangle_{C} \psi$. Then there exists a world $v$ in $\mathfrak{M}$ such that $(w, v) \in\left(R_{\varphi_{1}} \cup R_{\varphi_{2}}\right)^{*}$ and $\langle\mathfrak{M}, v\rangle \models \psi$. We distinguish the following two cases:

(a) $\mathfrak{M} \models \varphi_{1} \leftrightarrow \varphi_{2}$;

(b) $\langle\mathfrak{M}, u\rangle \models\left(\varphi_{1} \wedge \neg \varphi_{2}\right) \vee\left(\varphi_{2} \wedge \neg \varphi_{1}\right)$ for some world $u$.

For $(a)$, it is clear that $R_{\varphi_{1}}=R_{\varphi_{2}}=R_{\varphi_{1}} \cup R_{\varphi_{2}}$ and, as each $R_{\varphi_{i}}$ is reflexive and transitive, it holds that $\left(R_{\varphi_{1}} \cup R_{\varphi_{2}}\right)^{*}=R_{\varphi_{i}}$ for every $i \in\{1,2\}$. Hence, $(w, v) \in\left(R_{\varphi_{1}} \cup R_{\varphi_{2}}\right)^{*}$ implies that $(w, v) \in R_{\varphi_{i}}$ which yields $\langle\mathfrak{M}, w\rangle \models\left\langle\varphi_{i}\right\rangle_{K} \psi$ for every $i \in\{1,2\}$. Consequently, it holds that $\langle\mathfrak{M}, w\rangle \models\left\langle\varphi_{1}\right\rangle_{K} \psi \vee\left\langle\varphi_{2}\right\rangle_{K} \psi \vee\left\langle\varphi_{1}\right\rangle_{K}\left\langle\varphi_{2}\right\rangle_{K} \psi \vee\left\langle\varphi_{2}\right\rangle_{K}\left\langle\varphi_{1}\right\rangle_{K} \psi$.

For $(b)$, we show that the relation $R=\left(R_{\varphi_{1}} \cup R_{\varphi_{2}}\right) \circ\left(R_{\varphi_{1}} \cup R_{\varphi_{2}}\right)$ is a universal relation, where ' $O$ ' is the composition operation on relations. Let $t, s \in W$ be two worlds. We show that $(t, s) \in R$. We will use the world $u$ as a linking point between $s$ and $t$. We know that each $R_{\varphi_{i}}$ is of the form $\llbracket \varphi_{i} \rrbracket \otimes \llbracket \neg \varphi_{i} \rrbracket$ (similar to Example 3 if we take $p=\varphi_{1}$ and $q=\varphi_{2}$ ). Hence, since $\langle\mathfrak{M}, u\rangle \models\left(\varphi_{1} \wedge \neg \varphi_{2}\right) \vee\left(\varphi_{2} \wedge \neg \varphi_{1}\right)$, it will belong to the upper cluster according to the first relation among $R_{\varphi_{1}}$ and $R_{\varphi_{2}}$, while it will belong to the lower cluster according to the second relation. Therefore $(t, u) \in\left(R_{\varphi_{1}} \cup R_{\varphi_{2}}\right)$ and, similarly, $(u, s) \in\left(R_{\varphi_{1}} \cup R_{\varphi_{2}}\right)$. Hence, it holds that $(t, s) \in R$. Since $t$ and $s$ were chosen arbitrarily, we can infer that $R=W \times W$, and as $R$ is reflexive and transitive we have that $R^{*}=R$. Now $(w, v) \in\left(R_{\varphi_{1}} \cup R_{\varphi_{2}}\right)^{*}$ implies that $(w, v) \in R$. This means that the world $v$ can be reached from $w$ in two steps, i.e., it holds that $(w, v) \in\left(R_{\varphi_{1}} \circ R_{\varphi_{1}}\right),(w, v) \in\left(R_{\varphi_{2}} \circ R_{\varphi_{2}}\right),(w, v) \in\left(R_{\varphi_{2}} \circ R_{\varphi_{1}}\right)$ or $(w, v) \in\left(R_{\varphi_{1}} \circ R_{\varphi_{2}}\right)$. We obtain the first two since each $R_{\varphi_{i}}$ is transitive, which means that $v$ can be reached in one step as well, whereas the second two cases do not simplify further. For each of the above case it holds that $\langle\mathfrak{M}, w\rangle \models\left\langle\varphi_{1}\right\rangle_{K} \psi \vee\left\langle\varphi_{2}\right\rangle_{K} \psi \vee\left\langle\varphi_{1}\right\rangle_{K}\left\langle\varphi_{2}\right\rangle_{K} \psi \vee\left\langle\varphi_{2}\right\rangle_{K}\left\langle\varphi_{1}\right\rangle_{K} \psi$.

For the other direction from right to left, suppose that $\langle\mathfrak{M}, w\rangle \not \models\left\langle\left\{\varphi_{1}, \varphi_{2}\right\}\right\rangle_{C} \psi$. Then, it holds that $\langle\mathfrak{M}, w\rangle \models\left[\left\{\varphi_{1}, \varphi_{2}\right\}\right]_{C} \neg \psi$. By Proposition 3 we infer that $\langle\mathfrak{M}, w\rangle \mid=\left[\varphi_{1}\right]_{K} \neg \psi \wedge$ $\left[\varphi_{2}\right]_{K} \neg \psi \wedge\left[\varphi_{1}\right]_{K}\left[\varphi_{2}\right]_{K} \neg \psi \wedge\left[\varphi_{2}\right]_{K}\left[\varphi_{1}\right]_{K} \neg \psi$. As a result we obtain that $\langle\mathfrak{M}, w\rangle \not \models\left\langle\varphi_{1}\right\rangle_{K} \psi \vee$ $\left\langle\varphi_{2}\right\rangle_{K} \psi \vee\left\langle\varphi_{1}\right\rangle_{K}\left\langle\varphi_{2}\right\rangle_{K} \psi \vee\left\langle\varphi_{2}\right\rangle_{K}\left\langle\varphi_{1}\right\rangle_{K} \psi$.

Lemma 1. Let $\varphi_{1}$ and $\varphi_{2}$ be two formulas in the language of $S 5^{r}$. Then the formula $\left[\varphi_{1}, \varphi_{2}\right]_{C} p \leftrightarrow$ $\psi(p)$ with $\psi(p)=\left[\varphi_{1}\right]_{K} p \wedge\left[\varphi_{2}\right]_{K} p \wedge\left[\varphi_{1}\right]_{K}\left[\varphi_{2}\right]_{K} p \wedge\left[\varphi_{2}\right]_{K}\left[\varphi_{1}\right]_{K} p$ is a relational modal definition for the language of $S 5^{r}$.

Proof. We need to show the existence of a first-order formula $\Psi_{\varphi_{1}, \varphi_{2}}(x, y)$ with two free variables $x$ and $y$ that uses only predicates from $\operatorname{ST}_{x}(\psi(p))$ such that for every $\chi \in \mathrm{S}^{r}$ and every pointed model $\langle\mathfrak{M}, w\rangle$, it holds that $\langle\mathfrak{M}, w\rangle \models(\forall y)\left(\Psi_{+}(x, y) \Rightarrow \operatorname{ST}_{y}(\psi)\right)$ iff $\mathfrak{M} \models \operatorname{ST}_{x}(\psi(\chi))[x \mapsto$ $c_{w}$ ] (cf. Definition 1). 
Let $\Psi_{\varphi_{1}, \varphi_{2}}(x, y)$ be such that $(v, w) \in R_{\left\{\varphi_{1}, \varphi_{2}\right\}}$ iff $\mathfrak{M} \models \Psi_{\varphi_{1}, \varphi_{2}}(x, y)\left[x \mapsto c_{v}, y \mapsto c_{w}\right]$, where

$$
R_{\left\{\varphi_{1}, \varphi_{2}\right\}}=\left(R_{\varphi_{1}} \cup R_{\varphi_{2}}\right) \circ\left(R_{\varphi_{1}} \cup R_{\varphi_{2}}\right) .
$$

Moreover, let $\mathfrak{M}$ be a model and let $w$ be a world in $\mathfrak{M}$. It suffices to show the following equivalence: $\mathfrak{M}=\left((\forall y)\left(R_{\left\{\varphi_{1}, \varphi_{2}\right\}}(x, y) \Rightarrow P(y)\right)\right)\left[x \mapsto c_{w}\right]$ iff the following four conditions are satisfied:

- $\mathfrak{M} \models\left((\forall y)\left(R_{\varphi_{1}}(x, y) \Rightarrow P(y)\right)\right)\left[x \mapsto c_{w}\right] ;$

- $\mathfrak{M} \models\left((\forall y)\left(R_{\varphi_{2}}(x, y) \Rightarrow P(y)\right)\right)\left[x \mapsto c_{w}\right]$;

- $\mathfrak{M} \models\left((\forall y, z)\left(\left(R_{\varphi_{1}}(x, z) \wedge R_{\varphi_{2}}(z, y)\right) \Rightarrow P(y)\right)\right)\left[x \mapsto c_{w}\right]$; and

- $\mathfrak{M} \models\left((\forall y, z)\left(\left(R_{\varphi_{2}}(x, z) \wedge R_{\varphi_{1}}(z, y)\right) \Rightarrow P(y)\right)\right)\left[x \mapsto c_{w}\right]$,

where $\left[x \mapsto c_{w}\right]$ substitutes the occurrences of the free variable $x$ with the constant $c_{w}$ which is interpreted as $w$ in $\mathfrak{M}$ extended with dedicated constants, one for every world. The equivalence is a direct application of the following equality

$$
\left(R_{\varphi_{1}} \cup R_{\varphi_{2}}\right) \circ\left(R_{\varphi_{1}} \cup R_{\varphi_{2}}\right)=R_{\varphi_{1}} \cup R_{\varphi_{2}} \cup\left(R_{\varphi_{2}} \circ R_{\varphi_{1}}\right) \cup\left(R_{\varphi_{1}} \circ R_{\varphi_{2}}\right) .
$$

It suffices to show that $R_{\varphi_{1}} \cup R_{\varphi_{2}} \subseteq\left(R_{\varphi_{2}} \circ R_{\varphi_{1}}\right) \cup\left(R_{\varphi_{1}} \circ R_{\varphi_{2}}\right)$. But this is immediate since each $R_{\varphi}$ is a reflexive relation.

The reduction of the common knowledge modality for a finite set $\Phi=\left\{\varphi_{1}, \ldots, \varphi_{n}\right\}$ of agents is recursively defined as a function $\mathrm{Rd}$ as follows, where $n \geq 3$ :

$$
\begin{aligned}
& \operatorname{Rd}\left(\left[\varphi_{1}\right]_{C} p\right):= {\left[\varphi_{1}\right]_{K} p } \\
& \operatorname{Rd}\left(\left[\varphi_{1}, \varphi_{2}\right]_{C} p\right):=\left[\varphi_{1}\right]_{K} p \wedge\left[\varphi_{2}\right]_{K} p \wedge\left[\varphi_{1}\right]_{K}\left[\varphi_{2}\right]_{K} p \wedge \\
& \\
& \operatorname{Rd}\left(\left[\varphi_{1}, \ldots, \varphi_{n}\right]_{C} p\right):=\operatorname{Rd}\left(\left[\operatorname{Rd}\left(\left[\varphi_{1}, \ldots, \varphi_{n-1}\right]_{C} p\right), \varphi_{n}\right]_{C} p\right)
\end{aligned}
$$

Note that here we implicitly assume that each $\varphi_{i}$ belongs to the language of $\mathrm{S} 5^{r}$, i.e., $\varphi_{i}$ does not contain the modality for common knowledge. The reduction for arbitrary formulas $\Phi=\left\{\varphi_{1}, \ldots, \varphi_{n}\right\}$, where the $\varphi_{i}$-s may include the common knowledge modality, is a simple application of the reduction step by step. It can readily be seen that the function Rd yields a formula in the language of $\mathrm{S}^{r}$.

Theorem 3. Let $\Phi$ be a finite set of formulas in the language of $\mathrm{S5}^{r}$. Then the formula $[\Phi]_{C} p \leftrightarrow R d\left([\Phi]_{C} p\right)$ is a relational modal definition for the language of $S^{r}$.

Proof. The case where $|\Phi|=1$ is trivial; and the case where $|\Phi|=2$ follows from Lemma 1. Let $\Phi=\left\{\varphi_{1}, \ldots, \varphi_{n}\right\}$ with $n \geq 3$. We show that $\left[\varphi_{1}, \ldots, \varphi_{n}\right]_{C} p \leftrightarrow \operatorname{Rd}\left(\left[\varphi_{1}, \ldots, \varphi_{n}\right]_{C} p\right)$ is a relational modal definition. It holds that

$$
\operatorname{Rd}\left(\left[\varphi_{1}, \ldots, \varphi_{n}\right]_{C} p\right)=\operatorname{Rd}\left(\left[\operatorname{Rd}\left(\left[\varphi_{1}, \ldots, \varphi_{n-1}\right]_{C} p\right), \varphi_{n}\right]_{C} p\right) .
$$

Let $\gamma=\operatorname{Rd}\left(\left[\varphi_{1}, \ldots, \varphi_{n-1}\right]_{C} p\right)$. It can readily be seen that $\gamma$ is a formula in the language $\mathrm{S}^{r}$. Then $\operatorname{Rd}\left(\left[\varphi_{1}, \ldots, \varphi_{n}\right]_{C} p\right) \leftrightarrow \operatorname{Rd}\left(\left[\gamma, \varphi_{n}\right]_{C} p\right)$ and by Lemma 1 is equivalent to $[\gamma]_{K} p \wedge\left[\varphi_{2}\right]_{K} p \wedge$ $[\gamma]_{K}\left[\varphi_{2}\right]_{K} p \wedge\left[\varphi_{2}\right]_{K}[\gamma]_{K} p$. Again by Lemma 1, it is a relational modal definition.

Let $\mathrm{S} \mathrm{C}^{r}$ be the logic obtained by extending $\mathrm{S}^{r}$ with modal definitions of the form $[\Phi]_{C} p \leftrightarrow$ $\operatorname{Rd}\left([\Phi]_{C} p\right)$, where $\Phi$ ranges over sets of formulas in the language of $\mathrm{S}^{r}$ and the function $\mathrm{Rd}$ is defined as above. We obtain the following result. 
Theorem 4. The modal logic S5C ${ }^{r}$ is sound and complete w.r.t. the class of all basic structures.

The proof of the theorem follows from theorems 1,2 and 3 .

Theorem 5. The logic S5C $\mathrm{C}^{r}$ is decidable.

Proof. Proof follows from decidability of the logic $S 5^{r}[13]$ and reducibility of an arbitrary S5C ${ }^{r}$ formula to a $\mathrm{S}^{r}$ formula.

\section{Conclusion}

In this paper, we investigating the logic $\mathrm{S}^{2} \mathrm{C}^{r}$ for which we prove Kripke completeness and decidability results. Possible directions for future work are to investigate extension of $\mathrm{S}^{r}$ with distributed knowledge in more detail and axiomatize it and prove similar completeness theorems as for the logics $\mathrm{S}^{r}, \mathrm{~S}^{2} \mathrm{C}^{r}$.

\section{References}

[1] Jon Barwise. Three views of common knowledge. In Horacio Arló-Costa, Vincent F. Hendricks, and Johan van Benthem, editors, Readings in Formal Epistemology: Sourcebook, pages 759-772. Springer International Publishing, Cham, 2016.

[2] Guram Bezhanishvili, Leo Esakia, and David Gabelaia. Some results on modal axiomatization and definability for topological spaces. Studia Logica, 81(3):325-355, Dec 2005.

[3] Nick Bezhanishvili and Wiebe van der Hoek. Structures for epistemic logic. To appear in Logical and Informational Dynamics, a volume in honour of Johan van Benthem, 2014.

[4] P. Blackburn, M. de Rijke, and Y. Venema. Modal Logic. Cambridge University Press, Cambridge, UK, 2001.

[5] Brian F. Chellas. Basic conditional logic. Journal of Philosophical Logic, 4:133-153, 1975.

[6] Brian F. Chellas. Modal Logic: An Introduction. Cambridge University Press, 1980.

[7] Leo Esakia. Around provability logic. Annals of Pure and Applied Logic, 161(2):174-184, 2009. Festschrift on the occasion of Franco Montagna's 60th birthday.

[8] Andreas Herzig, Tiago de Lima, and Emiliano Lorini. On the dynamics of institutional agreements. Synthese, 171(2):321-355, Nov 2009.

[9] David Lewis. Convention: A Philosophical Study. Harvard University Press, Cambridge, Massachusetts, 1969.

[10] David Lewis. Counterfactuals. Harvard University Press, Cambridge, Massachusetts, 1973. revised printing Blackwell.

[11] Jan A. Plaza. Logics of public communications. In Proceedings of the 4th International Symposium on Methodologies for Intelligent Systems, pages 201-216. Oak Ridge National Laboratory, ORNL/DSRD-24, 1989.

[12] Robert Stalnaker. A theory of conditionals. Studies in Logical Theory, pages 98-112, 1968.

[13] Levan Uridia and Dirk Walther. An epistemic logic with hypotheses. In Hans van Ditmarsch, Jérôme Lang, and Shier Ju, editors, Logic, Rationality, and Interaction, pages 286-299, Berlin, Heidelberg, 2011. Springer Berlin Heidelberg.

[14] Levan Uridia and Dirk Walther. Completeness by modal definitions. In Guillermo R. Simari, Eduardo Fermé, Flabio Gutiérrez Segura, and José Antonio Rodríguez Melquiades, editors, Advances in Artificial Intelligence - IBERAMIA 2018, pages 67-79, Cham, 2018. Springer International Publishing. 
[15] Johann van Benthem. Rational dynamics and epistemic logic in games. International Game Theory Review, 9:13-45, 2007. Erratum reprint, Volume 9:2, 377-409.

[16] Hans van Ditmarsch, Wiebe van der Hoek, and Barteld Kooi. Dynamic Epistemic Logic, volume 337 of Synthese Library: Studies in Epistemology, Logic, Methodology, and Philosophy of Science. Springer, Dordrecht, The Netherlands, 2007. 